\title{
Remyelination Is Altered by Bone Morphogenic Protein Signaling in Demyelinated Lesions
}

\author{
Jennifer K. Sabo, ${ }^{1,2}$ Tim D. Aumann, ${ }^{1,2}$ Daniel Merlo, ${ }^{2}$ Trevor J. Kilpatrick, ${ }^{1,2}$ and Holly S. Cate ${ }^{1,2}$ \\ ${ }^{1}$ Centre for Neuroscience and ${ }^{2}$ Florey Neuroscience Institutes, University of Melbourne, Parkville, Victoria 3010, Australia
}

Remyelination of the CNS involves the regeneration of mature oligodendrocytes by endogenous oligodendrocyte progenitor cells (OPCs). Previous studies have shown that bone morphogenic proteins (BMPs) inhibit the production of oligodendrocytes in the healthy CNS. However, there is currently no information on the influence of BMP signaling in vivo within demyelinated lesions of the brain or on subsequent remyelination. Here, we determine a role for BMP signaling in modulating oligodendrogliogenesis and remyelination in the brain following cuprizone-induced demyelination. We identified that BMP signaling is active in oligodendroglia and astrocytes within the demyelinated corpus callosum. Intraventricular infusion of BMP4 into the brains of mice during demyelination increased the proliferation of OPCs and, to a lesser extent, microglia and astrocytes in the corpus callosum. In contrast, infusion of Noggin, an extracellular antagonist of BMP4, increased the density of mature oligodendrocytes in the remyelinating corpus callosum. Additional evidence from myelin staining and electron microscopy indicates there is an increase in remyelinated axons in the corpus callosum of Noggininfused mice. Thus, inhibition of endogenous BMP signaling during demyelination promotes mature oligodendrocyte regeneration and remyelination.

\section{Introduction}

In demyelinating diseases of the CNS, axons lose their myelin sheaths and consequently are vulnerable to degeneration. Apoptosis of oligodendrocytes, the cells that produce the myelin sheaths, is a key process in CNS demyelination (Barnett and Prineas, 2004). Remyelination is a natural regenerative mechanism whereby myelin sheaths are restored to the damaged axons, protecting them from further degeneration (Irvine and Blakemore, 2008). Studies using genetic lineage tracing in transgenic mice indicate that oligodendrocyte progenitor cells (OPCs) are an endogenous source of mature, myelinating oligodendrocytes in the healthy adult CNS (Rivers et al., 2008) and that in animal models of demyelination resident OPCs generate remyelinating oligodendrocytes within lesions (Zawadzka et al., 2010). In Multiple Sclerosis, OPCs are present in lesions, but often remain quiescent (Wolswijk, 1998; Kuhlmann et al., 2008). Therefore, enhancing OPC differentiation is a promising strategy to promote remyelination in lesions.

Bone morphogenic proteins (BMPs) are secreted proteins that form the largest subclass of the transforming growth factor- $\beta$ superfamily of cytokines. BMP2 and BMP4, which comprise one subfamily of BMPs, bind to one of two type I signal-transducing receptors (BMPRIa and BMPRIb) and one type II ligand binding receptor (BMPRII) (Massagué, 1998). Phosphorylation of receptor-

\footnotetext{
Received Nov. 7, 2010; revised Dec. 21, 2010; accepted Jan. 29, 2011.

The National Health and Medical Research Council of Australia and Holloway Family Trust supported this work. A Multiple Sclerosis Research Australia Postgraduate Scholarship supported J.K.S. The University of Melbourne, William Collie Fellowship supported H.S.C. Anna Friedhuber provided technical assistance with electron microscopy. Charityworks for MS and the Victorian Operational Infrastructure Support Program provided additional support. The authors declare no competing financial interests.

Correspondence should be addressed to Holly S. Cate, Centre for Neuroscience, University of Melbourne, Parkville, Victoria 3010, Australia. E-mail: hcate@unimelb.edu.au.

DOI:10.1523/JNEUROSCI.5859-10.2011

Copyright $\odot 2011$ the authors $\quad 0270-6474 / 11 / 314504-07 \$ 15.00 / 0$
}

regulated SMAD1/5/8 is increased upon BMP ligand/receptor formation and is indicative of BMP pathway activation (Massagué, 1998). OPCs and oligodendrocytes derived from the CNS express BMP4 and all three BMP receptors (Kondo and Raff, 2004). Exogenous BMP4 inhibits OPC differentiation and promotes astroglial differentiation in vitro (Grinspan et al., 2000). Conversely, Noggin, an extracellular antagonist of BMP4 (Massagué, 1998), blocks the production of astrocytes by BMP4 when applied to OPC cultures (Sim et al., 2006). These studies suggest an inhibitory role for BMPs in OPC differentiation.

There is evidence that BMPs are regulated during demyelination. Within demyelinated lesions induced by ethidium bromide in the brain, BMP4 expression is increased in OPCs and is absent upon remyelination (Zhao et al., 2005). In an animal model of multiple sclerosis, experimental autoimmune encephalomyelitis, BMPs were upregulated in the demyelinated spinal cord, with BMP4 expression being most abundant and increasing with enhanced clinical disease severity (Ara et al., 2008). The cellular sources of BMP4 included macrophages, astrocytes and oligodendrocytes within the lesions (Ara et al., 2008). While these studies suggest a possible role for BMPs within demyelinating lesions, the function of BMP signaling in demyelination and remyelination was not examined.

In this study, we report the effects of modulating BMP signaling upon adult OPCs and astrocytes during demyelination and remyelination in vivo. We show that BMP signaling is active in OPCs and astrocytes within demyelinated lesions in the corpus callosum (CC). Infusion of BMP4 increased the proliferation of glial cells, predominantly OPCs, during demyelination. We further show that infusion of Noggin increased the numbers of mature oligodendrocytes and enhanced remyelination in the CC following cuprizone-induced demyelination. 


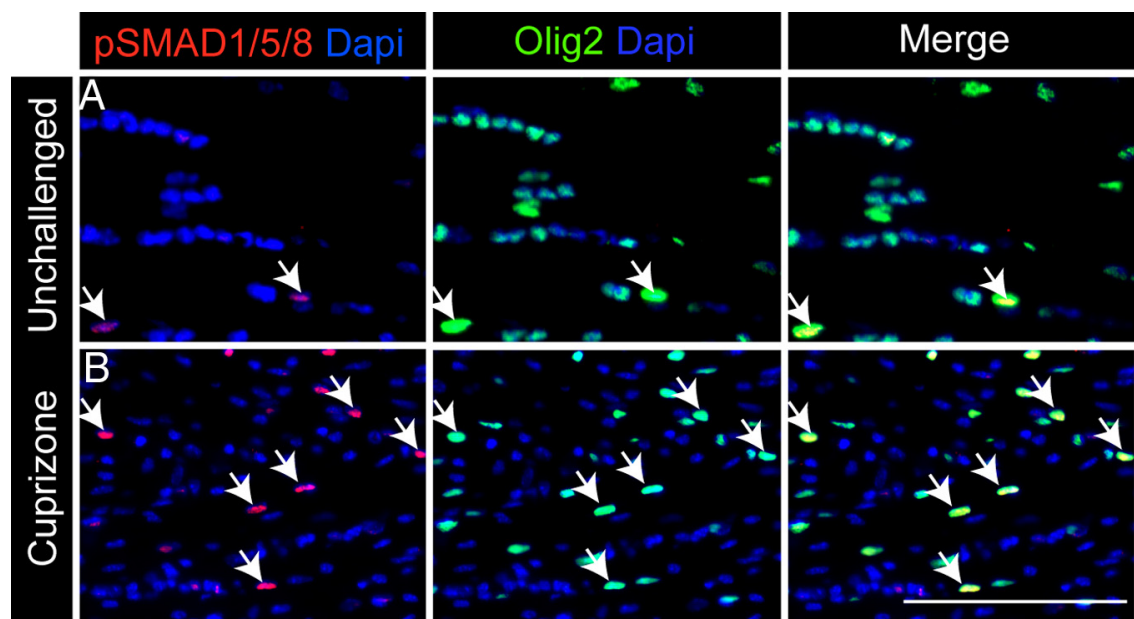

C
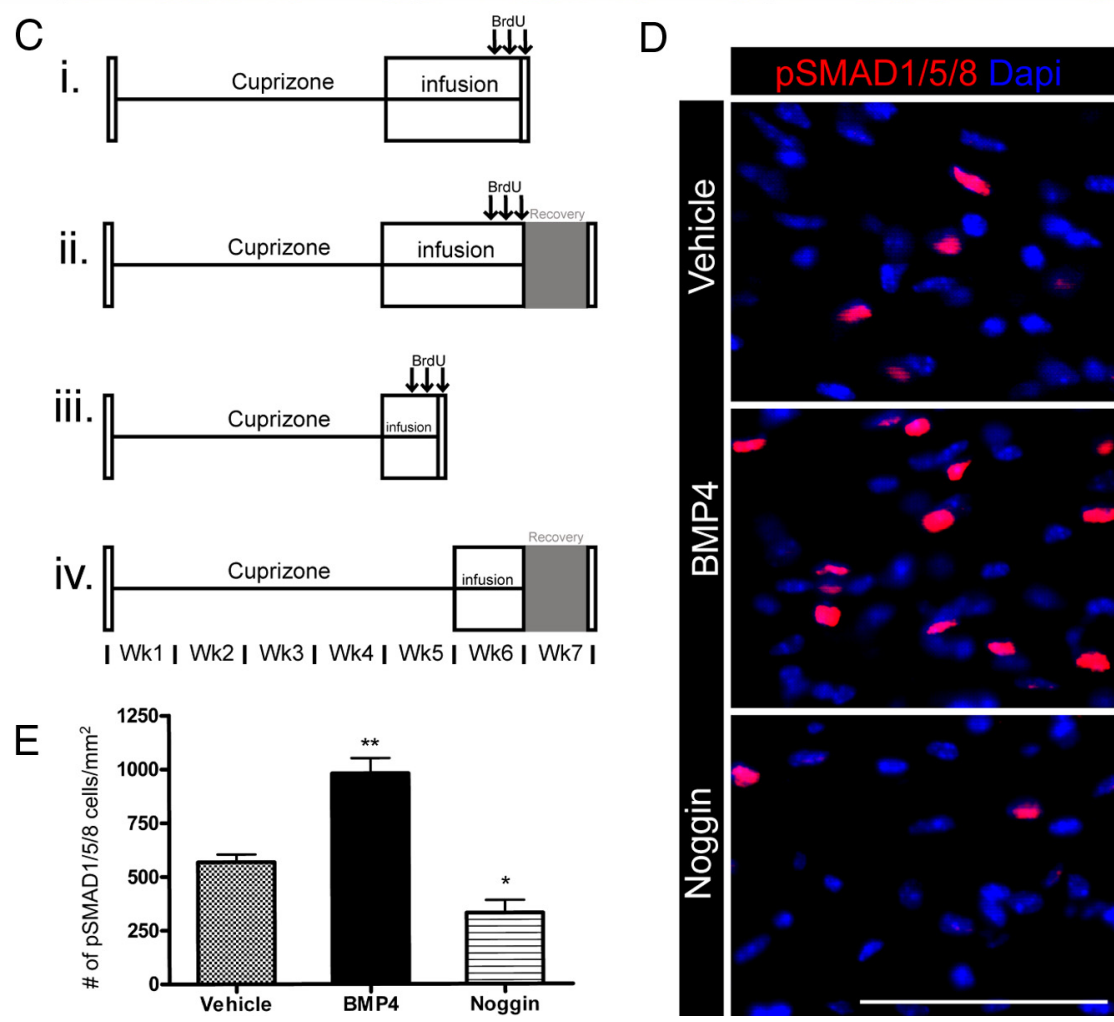

Figure 1. BMP signaling is active in oligodendroglial cells within demyelinating lesions. $A, B$, pSMAD1/5/8 and 0lig2 immunoreactivity in unchallenged and cuprizone-challenged mice. Merged images reveal colabeling of pSMAD1/5/8 and 0 lig 2 within a subset of cells in the $C C$ of unchallenged and 6 week cuprizone-challenged mice. Arrows indicate double-positive cells. C, Timelines $(\boldsymbol{i}$-iv) for infusion experiments detailed in Results. D, Immunostaining of pSMAD1/5/8 in the CC of $14 \mathrm{~d}$ infused mice after 6 weeks of cuprizone challenge. $\boldsymbol{E}$, Quantification of the density of PSMAD1/5/8 ${ }^{+}$cells in the $\mathrm{CC}$ of $14 \mathrm{~d}$ infused mice after 6 weeks of cuprizone challenge. $n=4-6$ animals per group. ${ }^{*} p<0.05,{ }^{* *} p<0.01$. Scale bars: $\boldsymbol{B}, \boldsymbol{D}, 50 \mu \mathrm{m}$.

\section{Materials and Methods}

Adult female C57BL/6 mice were used for all experiments according to guidelines previously described (Cate et al., 2010). Cuprizone-mediated demyelination was induced as previously described (Cate et al., 2010). For remyelination studies, mice were returned to normal chow for 1 week following cuprizone challenge. Recombinant human BMP4 or mouse Noggin (R\&D Systems) dissolved in artificial CSF (aCSF) at a dose of $400 \mathrm{ng}$ per day or aCSF was delivered into the lateral ventricle by mini-osmotic pumps (ALZET, Durect Corporation) (model 1002, $14 \mathrm{~d}$ infusion, $0.25 \mu \mathrm{l} / \mathrm{h}$ flow rate; model 1007, 5 and $7 \mathrm{~d}$ infusions, $0.50 \mu \mathrm{l} / \mathrm{h}$ flow rate) using Brain Infusion Kit III as previously described (Cate et al., 2010). Mice received $1 \mathrm{mg} / \mathrm{ml}$ 5-bromodeoxyuridine (BrdU) (Sigma-Aldrich) in their drinking water for $3 \mathrm{~d}$ during the infusion as indicated in the experimental timelines (Fig. 1C).

Brain tissue was collected, cryosectioned, and immunoprobed as previously described (Cate et al., 2010) with the following additional antibodies: BrdU (rat; AbD Serotec; 1:40), cleaved caspase-3 (rabbit; Cell Signaling Technology; 1:200), platelet-derived growth factor $\alpha$ receptor $(\mathrm{PDGFR} \alpha)$ (rat; eBioscience; 1:500), oligodendrocyte (clone RIP) (mouse; Millipore; $1: 10,000)$, and ionized calcium-binding adapter molecule 1 (IBA1) (rabbit; Wako Pure Chemicals; 1:1000), or prepared for electron microscopy using standard protocols. For fluorescent myelin staining, sections were incubated with FluoroMyelin (1:200; Invitrogen) for $1 \mathrm{~h}$ at room temperature.

All cell counts and area analyses were performed blind to experimental treatment. Immunopositive cells and area measures were quantified as previously described (Cate et al., 2010) and data are expressed as mean value/ $\mathrm{mm}^{2} \pm$ SEM. Images $(20 \times)$ were taken from three to six sections $50 \mu \mathrm{m}$ apart for each animal at locations between $-0.46 \mathrm{~mm}$ and -1.22 $\mathrm{mm}$ bregma with the midline CC at the epicenter and lateral borders determined by the image width. Myelinated axons quantification was performed on four $3000 \times$ images per animal. Diameter measures of $G$ ratios (the ratio of axon diameter to the axon plus myelin sheath diameter) were calculated using ImageJ software for at least 400 fibers per animal. For FluoroMyelin intensity analysis, images were converted to grayscale and the mean gray value of the midline $\mathrm{CC}$ was divided by the mean gray value of the dorsal fornix.

Statistical tests were performed using GraphPad Prism. One-way ANOVA with Dunnett's multiple-comparison test was used for comparison between groups in $14 \mathrm{~d}$ infusion experiments, two-way ANOVA with Bonferroni's post hoc test was used for distribution of $\mathrm{G}$ ratios, and Student's unpaired $t$ test was used for all other comparisons.

\section{Results}

\section{Demyelination within the corpus} callosum influences BMP signaling We first investigated whether BMP signaling is increased in the CC during demyelination. To undertake this analysis, we used the cuprizone-induced demyelination model. We first confirmed that 6 weeks of cuprizone challenge induced both demyelination within the CC (data not shown) and a significant decrease in the number of Olig2 oligodendroglia in the CC (unchallenged $1962 \pm 111.3 / \mathrm{mm}^{2}$; cuprizone $\left.685 \pm 81.4 / \mathrm{mm}^{2} ; p<0.0001\right)$. In addition to oligodendrocyte loss, there was recruitment of astrocytes and microglia/macrophages in the CC (data not shown).

After 6 weeks of cuprizone challenge, there was enhanced BMP signaling evidenced by an increased number of pSMAD1/ $5 / 8^{+}$cells (unchallenged, $109 \pm 9.28 / \mathrm{mm}^{2}$; cuprizone, $505 \pm$ $83.0 / \mathrm{mm}^{2} ; p<0.01$ ) (Fig. $1 A, B$ ). The majority of pSMAD1/5/8 cells colabeled with Olig2 in unchallenged $(91 \pm 4.2 \%)$ and 
cuprizone-challenged mice (54 $\pm 3.1 \%$ ) with a significant increase in pSMAD1/5/ $8^{+}$Olig ${ }^{+}$cells in cuprizone-challenged mice (unchallenged $97 \pm 9.8 / \mathrm{mm}^{2}$; cuprizone $\left.257 \pm 42.5 / \mathrm{mm}^{2} ; p<0.05\right)$. In addition, some pSMAD $1 / 5 / 8^{+}$cells expressed GFAP in unchallenged (4.4 \pm $1.6 \%)$ and cuprizone animals (21 \pm $6.6 \%)$. We found no $\mathrm{GFAP}^{+}$cells that expressed Olig2. To determine the substrate for future infusion experiments, pSMAD $1 / 5 / 8^{+}$cells were assessed at 4 weeks of cuprizone challenge in the CC and the subventricular zone (SVZ), and similar numbers of positive cells were present in the two locations (data not shown). Thus, BMP signaling is increased in cuprizone-induced demyelinated lesions and is active in the majority of oligodendroglial cells and in a subset of astrocytes.

\section{Modulation of BMP signaling during cuprizone challenge alters glial cell numbers}

To determine the consequences of BMP signaling modulation in the demyelinated CC, we implanted mini-osmotic pumps to deliver BMP4, Noggin or vehicle into the lateral ventricles for the final $14 \mathrm{~d}$ of a 6 week cuprizone challenge (Fig. 1Ci). Initially, we established that BMP4 infusion increased and Noggin decreased the number of $\mathrm{pSMAD} 1 / 5 / 8^{+}$cells in the CC compared with vehicle infusion (Fig. $1 D, E$ ). We also found that BMP4 infusion increased the density of $\mathrm{pS}$ MAD $1 / 5 / 8^{+}$Olig2 ${ }^{+}$cells $(p<0.05)$ (vehicle $169 \pm 36.0 / \mathrm{mm}^{2}$; BMP4 $334 \pm 42.3 /$ $\mathrm{mm}^{2}$; Noggin $95 \pm 54 / \mathrm{mm}^{2}$ ).

We next examined the consequences of BMP4 and Noggin infusion on the proliferation of oligodendroglial cells during cuprizone challenge. When mice were assessed after 6 weeks of cuprizone challenge (Fig. $1 \mathrm{Ci}$ ), there was a significant increase in the density of both $\mathrm{BrdU}^{+}$and Olig2 ${ }^{+}$cells in the CC after $\mathrm{BMP} 4$ infusion $\left(\mathrm{BrdU}^{+}\right.$: vehicle $351 \pm$ $77.5 / \mathrm{mm}^{2}$; BMP4 $856 \pm 123 / \mathrm{mm}^{2} ; p<$ 0.01 ; Olig ${ }^{+}$: vehicle $446 \pm 35.0 / \mathrm{mm}^{2}$; BMP4 $\left.858 \pm 101 / \mathrm{mm}^{2} ; p<0.01\right)$. Noggin infusion did not alter the density of $\mathrm{BrdU}^{+}$or $\mathrm{Olig} 2^{+}$cells. In addition, BMP4-infused mice had increased $\mathrm{BrdU}^{+}$Olig2 ${ }^{+}$double-labeled cells compared with vehicleinfused mice, while there was no difference in Noggin-infused mice (Fig. $2 A, E$ ). There was no difference in the density of $\mathrm{GFAP}^{+}$cells in the $\mathrm{CC}$ among the three groups of mice (Fig. 2C,F).

To assess the consequences of BMP4 and Noggin infusion on recovery, we allowed an independent cohort of mice to recover for 1 week following a 6 week cuprizone challenge (Fig. 1Cii). We

E bar, $50 \mu \mathrm{m}$.
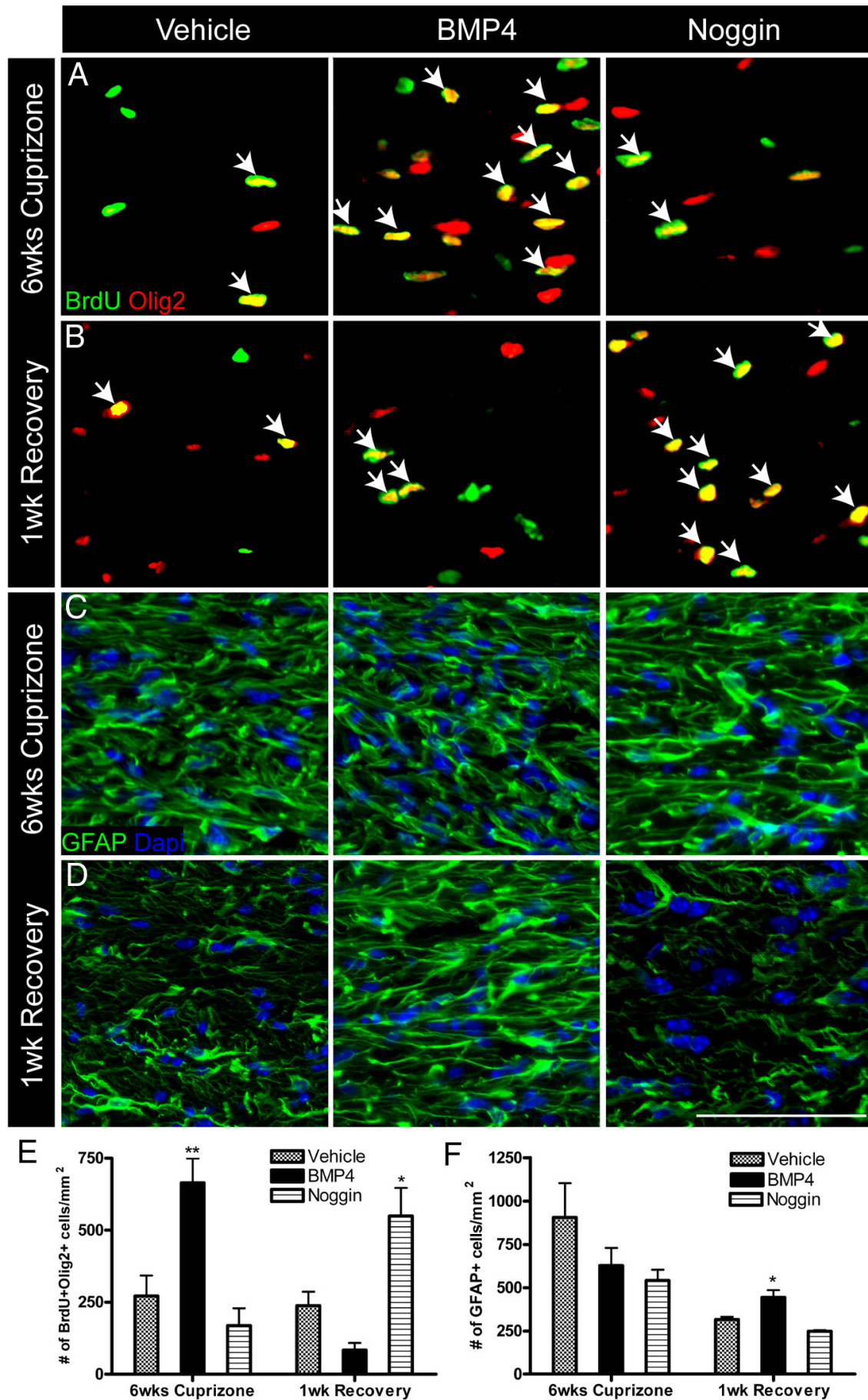

Figure 2. Modulation of BMP signaling during demyelination alters numbers of proliferating oligodendroglia and astrocytes A-D, Immunostaining of BrdU-0lig2 and GFAP in the CC of 14 d infused mice after 6 weeks of cuprizone challenge and 1 week of recovery. Arrows indicate double-positive cells. $E, F$, Quantification of the density of BrdU ${ }^{+} 0$ lig2 ${ }^{+}$cells and GFAP ${ }^{+}$cells in the CC of 14 dinfused mice after 6 weeks of cuprizone and 1 week of recovery. $n=4-6$ animals per group. ${ }^{*} p<0.05,{ }^{* *} p<0.01$. Scale

identified a significant increase in the density of $\mathrm{BrdU}^{+}$and Olig2 ${ }^{+}$cells in the Noggin-infused mice compared with vehicle $\left(\mathrm{BrdU}^{+}\right.$: vehicle $430 \pm 55.9 / \mathrm{mm}^{2} ;$ Noggin $741 \pm 83.9 / \mathrm{mm}^{2} ; p<$ 0.05 ; Olig $2^{+}$: vehicle $632 \pm 147 / \mathrm{mm}^{2}$; Noggin $1081 \pm 30.79 /$ $\left.\mathrm{mm}^{2} ; p<0.05\right)$. There was no significant difference in the density of BrdU ${ }^{+}$and Olig2 ${ }^{+}$cells between BMP4 and vehicle. However, we found an increase in the density of $\mathrm{BrdU}^{+} \mathrm{Olig} 2^{+}$doublelabeled cells in the Noggin-infused mice (Fig. $2 B, E$ ). To deter- 
mine whether the reduction of $\mathrm{BrdU}^{+}$Olig2 ${ }^{+}$double-labeled cells (between assessments at 6 weeks of cuprizone and 1 week recovery) in the BMP4-infused mice was due to migration of these cells from the midline to the lateral CC, we extended the analysis to the lateral CC at 1 week recovery. However, we found no difference in the density of $\mathrm{BrdU}^{+} \mathrm{Olig} 2^{+}$doublelabeled cells in the lateral CC (vehicle $169 \pm 42.8 / \mathrm{mm}^{2}$; BMP4 $99 \pm 24 / \mathrm{mm}^{2} ;$ Noggin $\left.244 \pm 21.7 / \mathrm{mm}^{2} ; p=0.12\right)$. When we examined astrocytes at 1 week of recovery, we found an increase in the density of GFAP ${ }^{+}$cells in the BMP4-infused mice within the CC, while there was no change in the Noggininfused mice (Fig. 2D,F). Together, these data indicate that BMP4 infusion during demyelination increases OPC numbers, however, following recovery from cuprizone challenge, these BrdU-positive cells are decreased and astrocyte numbers are increased. The data suggest that Noggin infusion during demyelination results in an increase in oligodendroglia during the recovery phase.

Short-term infusion of BMP4 during demyelination increases proliferation of glial cells

Given the increased proliferation following 2 weeks of BMP4 infusion, we investigated whether short-term BMP4 infusion would have similar consequences. To expedite this, mini-osmotic pumps were implanted after 4 weeks of cuprizone challenge, and mice were examined after a $5 \mathrm{~d}$ infusion with continued cuprizone challenge (Fig. 1Ciii). Short-term infusion of BMP4 increased BMP signaling as determined by pSMAD1/5/8 immunostaining (vehicle $323 \pm 42.9 / \mathrm{mm}^{2}$; BMP4 $563 \pm 70.7 / \mathrm{mm}^{2}$; $p<0.05)$ and increased the density of pSMAD $1 / 5 / 8^{+}$Olig $2^{+}$ cells (vehicle $128 \pm 21.8 / \mathrm{mm}^{2} ;$ BMP4 $326 \pm 56.2 / \mathrm{mm}^{2} ; p<$ 0.05). Consistent with the $14 \mathrm{~d}$ BMP4 infusion, we found a significant increase in the density of $\mathrm{BrdU}^{+} \mathrm{Olig} 2^{+}$doublelabeled cells compared with vehicle infusion (Fig. $3 A, D$ ). Characterization of these Olig2 cells revealed they were of the oligodendroglial lineage and all either expressed CC1, a mature oligodendrocyte marker, or PDGFR $\alpha$, a marker for OPCs. There was a significant increase in Olig ${ }^{+} \mathrm{PDGFR} \alpha{ }^{+}$cells in BMP4-infused mice (vehicle $97 \pm 8.9 / \mathrm{mm}^{2}$; BMP4 $309 \pm$ $\left.74.3 / \mathrm{mm}^{2} ; p<0.05\right)$.

Following short-term BMP4 infusion, the Olig2 ${ }^{+}$cells represented $54 \pm 7.9 \%$ of the $\mathrm{BrdU}^{+}$population, in contrast to $14 \mathrm{~d}$ BMP4 infusion where $80 \pm 7.3 \%$ of $\mathrm{BrdU}^{+}$cells were Olig2 ${ }^{+}$. Therefore, we examined the proliferation of astroglia and microglia in the CC of short-term infused animals. We found a significant increase in the density of $\mathrm{BrdU}^{+} \mathrm{IBA}^{+}$and $\mathrm{BrdU}^{+} \mathrm{GFAP}^{+}$ cells (Fig. 3B-D). BMP4 infusion significantly increased the density of $\mathrm{IBA}^{+}{ }^{+}$microglia/macrophages (vehicle $1278 \pm 175.3 /$ $\mathrm{mm}^{2}$; BMP4 $\left.2762 \pm 204.9 / \mathrm{mm}^{2} ; p<0.01\right)$, while the density of $\mathrm{GFAP}^{+}$cells was unchanged (vehicle $254 \pm 15.5 / \mathrm{mm}^{2}$; BMP4 $313 \pm 45.5 / \mathrm{mm}^{2} ; p=0.26$ ). Thus, short-term BMP4 infusion increased the proliferation of glial cells during cuprizone-induced demyelination.

In addition, we performed caspase- 3 immunostaining in the brains of mice subjected to short-term infusion. We found that there was a strong trend toward an increase in caspase- $3^{+}$cells in the CC of BMP4-infused mice $(p=0.09)$ (vehicle $3.6 \pm 0.8 /$ $\mathrm{mm}^{2}$; BMP4 $29 \pm 11 / \mathrm{mm}^{2}$; Noggin $8.7 \pm 2.9 / \mathrm{mm}^{2}$ ). These data would suggest that exogenously administered BMP4 is inducing apoptosis in this model.

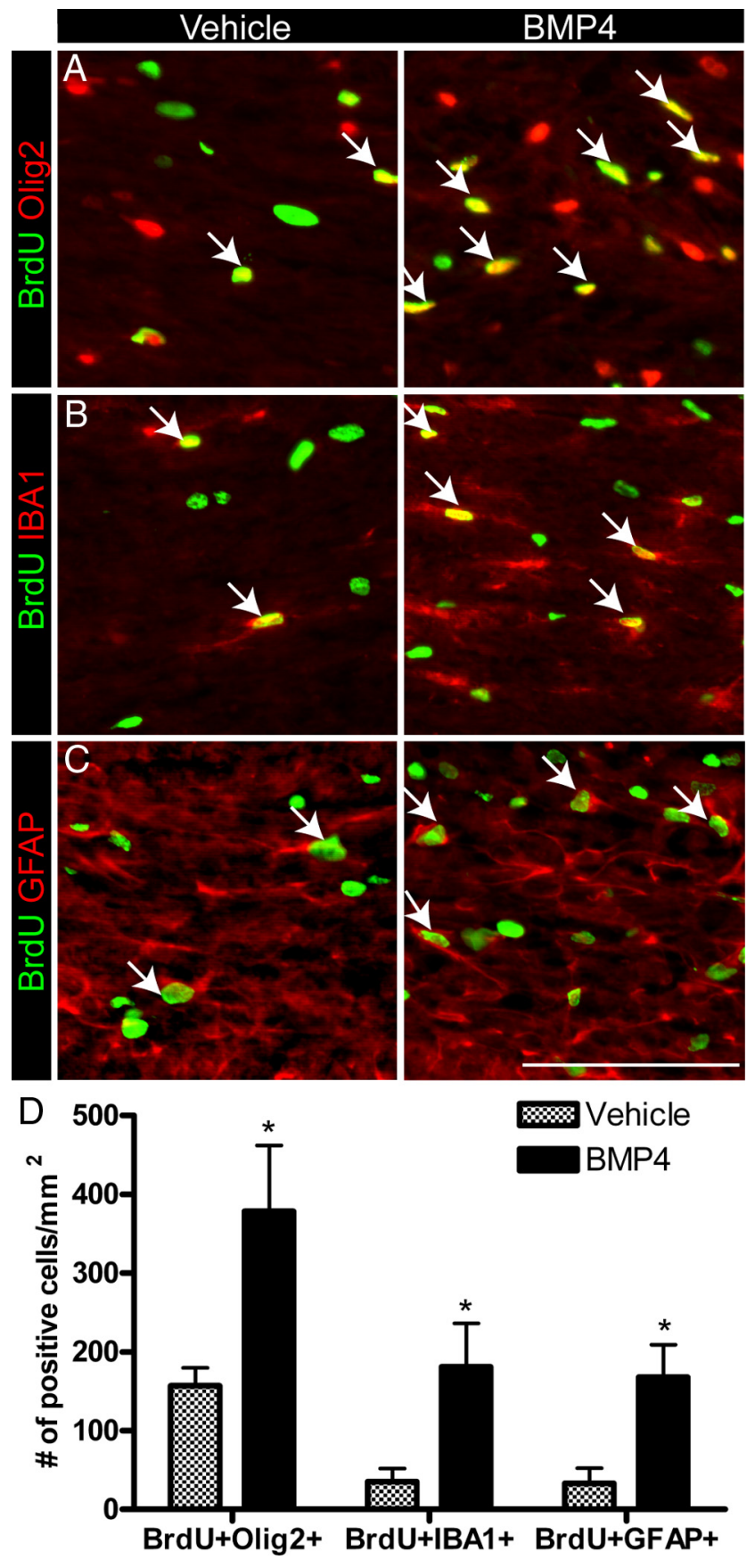

Figure 3. Short-term infusion of BMP4 during demyelination increases the proliferation of $O P C$, microglia and astrocytes. $\boldsymbol{A}-\boldsymbol{D}$. Immunostaining and quantification of the density of $\mathrm{BrdU}^{+}$Olig2 ${ }^{+}, \mathrm{BrdU}^{+} \mathrm{IBA} 1^{+}$, and BrdU ${ }^{+} \mathrm{GFAP}^{+}$cells in the $\mathrm{CC}$ after $5 \mathrm{~d}$ of either vehicle or BMP4 infusion. Arrows indicate double-positive cells. $n=4-5$ animals per group. ${ }^{*} p<0.05$. Scale bar, $50 \mu \mathrm{m}$.

Noggin infusion during demyelination results in an increase in the number of mature oligodendrocytes in the corpus callosum

Given that exogenous delivery of Noggin for $14 \mathrm{~d}$ resulted in an increase in the density of Olig ${ }^{+}$cells at 1 week of recovery, we next examined whether there was an increase in mature oligodendrocytes in these mice. We found that Noggin infusion significantly increased the density of mature oligodendrocytes that expressed both Olig2 and CC1 at 1 week of recovery (Fig. 4A,B). We also evaluated the numbers of cells with BrdU incorporation 
that differentiated into oligodendrocytes. $\mathrm{BrdU}^{+} \mathrm{CCl}^{+}$cells were significantly increased in Noggin-infused mice compared with vehicle $(p<0.05)$ (vehicle $69 \pm 23 / \mathrm{mm}^{2}$; Noggin $162 \pm 17.1 / \mathrm{mm}^{2}$; BMP4 $9 \pm 5 / \mathrm{mm}^{2}$ ). Thus, Noggin infusion during demyelination results in an increase in the number of mature oligodendrocytes during recovery from cuprizone challenge.

Noggin infusion during demyelination enhances remyelination in the corpus callosum

For analysis of myelination, we first performed immunostaining with the RIP antibody, which labels the myelin protein, $2^{\prime}, 3^{\prime}$-cyclic nucleotide $3^{\prime}$-phosphodiesterase (CNPase) (Watanabe et al., 2006). At 6 weeks of cuprizone, the three groups of $14 \mathrm{~d}$ infused mice displayed similar levels of RIP immunostaining (Fig. 4C). To further analyze myelination within the $\mathrm{CC}$, we quantified FluoroMyelin intensity, which revealed no significant difference in the infused mice (vehicle: $0.31 \pm 0.05$; BMP4: $0.36 \pm 0.01$; Noggin: $0.34 \pm 0.02$; $p=0.56)$. In contrast, after 1 week of recovery, we found that the intensity of RIP immunostaining was greater in vehicle- and Noggin-infused animals than in BMP4infused mice (Fig. 4C). On the other hand, at the 1 week recovery time point, FluoroMyelin intensity was significantly greater among Noggin-infused as opposed to vehicle-infused mice (Fig. $4 D$ ).

We further interrogated the effects of Noggin on remyelination by performing an additional infusion experiment with vehicle or Noggin infusion for the final $7 \mathrm{~d}$ of a 6 week cuprizone challenge followed by 1 week of recovery (Fig. 1Civ). There was an increased number of Olig ${ }^{+} \mathrm{CC}^{+}$ cells in Noggin-infused mice (vehicle $584 \pm 99.7 / \mathrm{mm}^{2}$; Noggin $1066 \pm 83.00 /$ $\left.\mathrm{mm}^{2} ; p<0.05\right)$, which is consistent with the $14 \mathrm{~d}$ infusion experiment. We next examined the ultrastructure of the caudal $\mathrm{CC}$ by electron microscopy and found an increase in the density of myelinated axons in the Noggin-infused mice (Fig. $4 E, G)$. In addition, the average $G$ ratio of the myelinated axons was increased in the Noggin-infused mice compared with vehicle (Fig. $4 F$ ), suggesting an increase in thinly myelinated axons. We also assessed the distribution of $\mathrm{G}$ ratios among the myelinated axons of the vehicle-infused mice and the three Noggin-infused mice that had increased numbers of myelinated axons. There was a similar number of axons with low $G$ ratios from vehicle- and Noggin-infused mice; however, in Noggin-infused mice, there was an increase in the number of axons with high $G$ ratios, which are (in $A) A, C, 50 \mu \mathrm{m} ; G, 1 \mu \mathrm{m}$.
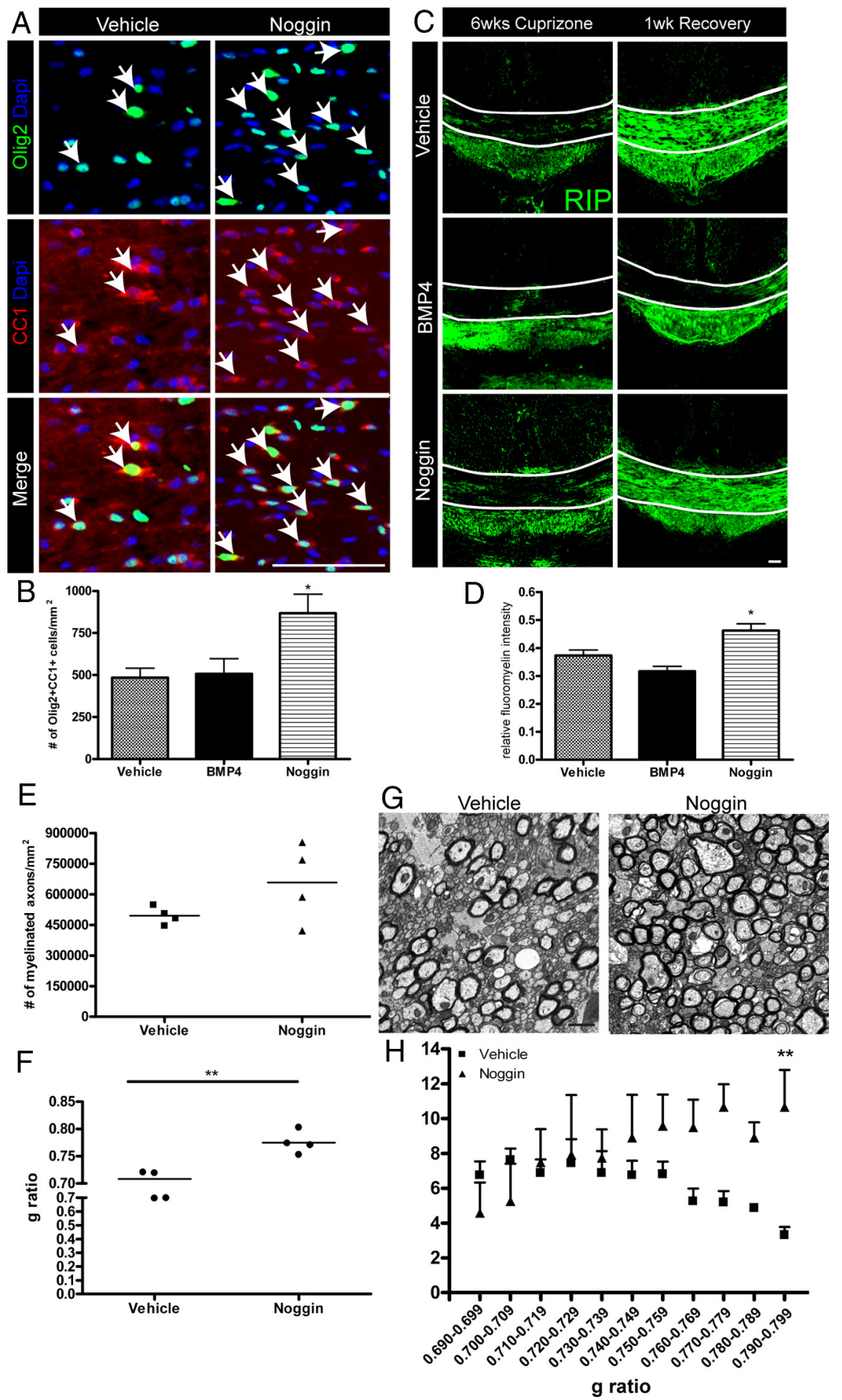

Figure 4. Noggin-infused mice have increased numbers of mature oligodendrocytes and remyelinated axons following recovery from cuprizone-induced demyelination. $A, B, 0$ lig2-CC 1 immunostaining and quantification in the $C($ of $14 \mathrm{~d}$ infused mice after 1 week of recovery. Arrows indicate double-positive cells. $C$, Representative images of RIP immunostaining in the $C C$ of $14 \mathrm{~d}$ infused mice. Lines indicate dorsal and ventral borders of the CC. $D$, Quantification of the mean relative FluoroMyelin intensity in the $C($ of $14 \mathrm{~d}$ infused mice after 1 week of recovery. $\boldsymbol{E}-\boldsymbol{H}$, Ultrastructural analysis of the $C(\mathrm{C}$ of $\mathrm{d}$ vehicle- and Noggin-infused mice after 1 week of recovery. Quantification of the density $(\boldsymbol{E})$ and average $\boldsymbol{G}$ ratio $(\boldsymbol{F})$ of myelinated axons. $\boldsymbol{G}$, Representative electron micrographs of the caudal $\mathrm{CC}$. $\boldsymbol{H}$, Number of axons per $\mathrm{G}$ ratio value. $n=3-4$ animals per group. ${ }^{*} p<0.05$; ${ }^{* *} p<0.01$. Scale bar:

indicative of thinner myelin sheaths, a feature of remyelinated axons (Fig. $4 \mathrm{H}$ ). In conclusion, these results suggest that Noggin infusion enhances remyelination following cuprizoneinduced demyelination. 


\section{Discussion}

In this study, we identified that BMP signaling is active in OPCs and astrocytes within the demyelinated CC following cuprizone challenge. We found that BMP4 infusion increased the numbers of OPCs during demyelination. However, following recovery from cuprizone challenge, oligodendroglia are decreased and astrocyte numbers are increased in BMP4-infused mice. Infusion of the BMP4 antagonist, Noggin, increased the number of mature oligodendrocytes and remyelination following recovery. Collectively, these results suggest that downregulating endogenous BMP signaling during myelin injury could have a beneficial influence on repair given that Noggin infusion increased numbers of mature oligodendrocytes and enhanced remyelination.

There is evidence for a dichotomous role of BMPs in affecting cell proliferation. Prior in vitro studies have reported that BMPs decrease the proliferation of embryonic and adult neural precursor cells (for review, see Sabo et al., 2009). In contrast, BMPs promote the proliferation of rat primary osteoblastic cells (Selvamurugan et al., 2007). In addition, recent work from our laboratory has shown that exogenous BMP4 increased the proliferation of adult SVZ neural precursor cells under differentiative conditions in vitro (Cate et al., 2010). We show here for the first time that BMP4 infusion increased the proliferation of OPCs and, to a lesser extent, microglia and astrocytes in the demyelinated CC in vivo. These data would suggest that exogenous BMP signaling could play a favorable role during demyelination since BMP4 infusion increased the pool of OPCs within demyelinated lesions.

Interestingly, however, we found a decrease in oligodendroglia numbers by 1 week of recovery in BMP4-infused mice. A key question that emerges therefore is the fate of the $\mathrm{BrdU}^{+} \mathrm{Olig} 2^{+}$ cells in the BMP4-infused mice between 6 weeks of cuprizone challenge and 1 week of recovery. At 1 week of recovery, we observed a reduction in $\mathrm{BrdU}^{+}$cells and we found no BrdU ${ }^{+}$ cells that expressed GFAP, suggesting that the OPCs did not differentiate into astrocytes. Furthermore, our analysis of the lateral CC, demonstrating no change in the density of OPCs, suggests that the OPCs did not migrate from the midline CC. Rather, our data showing an increase in the density of caspase- $3^{+}$cells suggests that delivery of BMP4 promoted apoptosis in vivo, which could contribute to the lack of OPCs following recovery from cuprizone challenge.

Our results support a role for Noggin in promoting remyelination after central demyelination. Noggin expanded the population of oligodendroglia during recovery from cuprizoneinduced demyelination. In our experimental paradigm, BrdU was administered at the end of cuprizone challenge and, at 1 week of recovery, we observed an increase in the number of $\mathrm{BrdU}^{+}$ cells that expressed Olig2 and CC1 in the CC of Noggin-infused compared with vehicle-infused mice. Given that cuprizone challenge increased the number of pSMAD $1 / 5 / 8^{+}$cells in the CC, and that this population (present in the CC at the 4 week time point) is decreased with subsequent Noggin infusion, our study suggests that Noggin acts on cells resident in the CC. Thus, our data are consistent with there being enhanced differentiation of resident OPCs in Noggin-infused mice. Jablonska et al. (2010) found that infusion of Chordin, a BMP antagonist with slightly lower affinity for BMP4 than Noggin (Piccolo et al., 1996), induced the differentiation of DCX-expressing cells of the adult SVZ into oligodendrocytes in lysolecithin-demyelinated CC. Thus, the combined data suggest that both inhibitors of BMP signaling (i.e., Chordin and Noggin) can increase the production of oligodendrocytes in vivo in the context of demyelination and that infusion of these substances are likely to have composite effects within the SVZ and the demyelinated lesion.

Successful remyelination not only depends on OPC proliferation, but also requires OPC survival and differentiation into myelinating oligodendrocytes. At 6 weeks of cuprizone ingestion, there is endogenous OPC proliferation within the demyelinated $\mathrm{CC}$, which we observed in our vehicle-infused mice. Upon withdrawal of cuprizone from the diet of mice, OPCs differentiate into myelinating oligodendrocytes and remyelination occurs (Mason et al., 2000). We found augmented remyelination following Noggin infusion that could be due to increased OPC survival or differentiation into mature oligodendrocytes. Similarly, Izrael et al. (2007) observed increased myelination in vivo when they transplanted Noggin-treated human embryonic stem cellderived OPCs in myelin-deficient shiverer mice. While there are caveats to quantifying remyelination in the CC due to the small diameter of axons (Stidworthy et al., 2003), we provide evidence in Noggin-infused mice that there is an increase in the density of myelinated axons and a greater proportion with higher $\mathrm{G}$ ratios, which are indicative of thinner myelin sheaths, a feature of remyelinated axons. Therefore, our finding of an increase in thin myelinated axons in Noggin-infused mice could be attributed to an increase in the number of remyelinated axons compared with the vehicle-infused mice.

In summary, the results in our study indicate an important role for BMP signaling in modulating oligodendrogliogenesis and remyelination. We have shown that exogenous BMP signaling transiently increases the proliferation of glial cells that are rapidly cleared following recovery from a demyelinating insult. Furthermore, inhibiting endogenous BMP signaling during demyelination promoted mature oligodendrocyte regeneration and myelin repair. Given that BMPs are expressed in demyelinating lesions in humans (Deininger et al., 1995), our findings identify a potential therapeutic target to enhance repair from demyelinating disease.

\section{References}

Ara J, See J, Mamontov P, Hahn A, Bannerman P, Pleasure D, Grinspan JB (2008) Bone morphogenetic proteins 4, 6, and 7 are up-regulated in mouse spinal cord during experimental autoimmune encephalomyelitis. J Neurosci Res 86:125-135.

Barnett MH, Prineas JW (2004) Relapsing and remitting multiple sclerosis: pathology of the newly forming lesion. Ann Neurol 55:458-468.

Cate HS, Sabo JK, Merlo D, Kemper D, Aumann TD, Robinson J, Merson TD, Emery B, Perreau VM, Kilpatrick TJ (2010) Modulation of bone morphogenic protein signalling alters numbers of astrocytes and oligodendroglia in the subventricular zone during cuprizone-induced demyelination. J Neurochem 115:11-22.

Deininger M, Meyermann R, Schluesener H (1995) Detection of two transforming growth factor-beta-related morphogens, bone morphogenetic proteins- 4 and -5 , in RNA of multiple sclerosis and Creutzfeldt-Jakob disease lesions. Acta Neuropathol 90:76-79.

Grinspan JB, Edell E, Carpio DF, Beesley JS, Lavy L, Pleasure D, Golden JA (2000) Stage-specific effects of bone morphogenetic proteins on the oligodendrocyte lineage. J Neurobiol 43:1-17.

Irvine KA, Blakemore WF (2008) Remyelination protects axons from demyelination-associated axon degeneration. Brain 131:1464-1477.

Izrael M, Zhang P, Kaufman R, Shinder V, Ella R, Amit M, Itskovitz-Eldor J, Chebath J, Revel M (2007) Human oligodendrocytes derived from embryonic stem cells: effect of noggin on phenotypic differentiation in vitro and on myelination in vivo. Mol Cell Neurosci 34:310-323.

Jablonska B, Aguirre A, Raymond M, Szabo G, Kitabatake Y, Sailor KA, Ming GL, Song H, Gallo V (2010) Chordin-induced lineage plasticity of adult SVZ neuroblasts after demyelination. Nat Neurosci 13:541-550.

Kondo T, Raff MC (2004) A role for Noggin in the development of oligodendrocyte precursor cells. Dev Biol 267:242-251.

Kuhlmann T, Miron V, Cui Q, Wegner C, Antel J, Brück W (2008) Differ- 
entiation block of oligodendroglial progenitor cells as a cause for remyelination failure in chronic multiple sclerosis. Brain 131:1749-1758.

Mason JL, Jones JJ, Taniike M, Morell P, Suzuki K, Matsushima GK (2000) Mature oligodendrocyte apoptosis precedes IGF-1 production and oligodendrocyte progenitor accumulation and differentiation during demyelination/remyelination. J Neurosci Res 61:251-262.

Massagué J (1998) TGF- $\beta$ signal transduction. Annu Rev Biochem 67:753-791.

Piccolo S, Sasai Y, Lu B, De Robertis EM (1996) Dorsoventral patterning in Xenopus: inhibition of ventral signals by direct binding of Chordin to BMP4. Cell 86:589-598.

Rivers LE, Young KM, Rizzi M, Jamen F, Psachoulia K, Wade A, Kessaris N, Richardson WD (2008) PDGFRA/NG2 glia generate myelinating oligodendrocytes and piriform projection neurons in adult mice. Nat Neurosci 11:1392-1401.

Sabo JK, Kilpatrick TJ, Cate HS (2009) Effects of bone morphogenic proteins on neural precursor cells and regulation during central nervous system injury. Neurosignals 17:255-264.

Selvamurugan N, Kwok S, Vasilov A, Jefcoat SC, Partridge NC (2007) Effects of BMP-2 and pulsed electromagnetic field (PEMF) on rat primary osteoblastic cell proliferation and gene expression. J Orthop Res 25:1213-1220.

Sim FJ, Lang JK, Waldau B, Roy NS, Schwartz TE, Pilcher WH, Chandross KJ,
Natesan S, Merrill JE, Goldman SA (2006) Complementary patterns of gene expression by human oligodendrocyte progenitors and their environment predict determinants of progenitor maintenance and differentiation. Ann Neurol 59:763-779.

Stidworthy MF, Genoud S, Suter U, Mantei N, Franklin RJ (2003) Quantifying the early stages of remyelination following cuprizone-induced demyelination. Brain Pathol 13:329-339.

Watanabe M, Sakurai Y, Ichinose T, Aikawa Y, Kotani M, Itoh K (2006) Monoclonal antibody Rip specifically recognizes $2^{\prime}, 3^{\prime}$-cyclic nucleotide $3^{\prime}$-phosphodiesterase in oligodendrocytes. J Neurosci Res 84: $525-533$.

Wolswijk G (1998) Chronic stage multiple sclerosis lesions contain a relatively quiescent population of oligodendrocyte precursor cells. J Neurosci 18:601-609.

Zawadzka M, Rivers LE, Fancy SP, Zhao C, Tripathi R, Jamen F, Young K, Goncharevich A, Pohl H, Rizzi M, Rowitch DH, Kessaris N, Suter U, Richardson WD, Franklin RJ (2010) CNS-resident glial progenitor/ stem cells produce Schwann cells as well as oligodendrocytes during repair of CNS demyelination. Cell Stem Cell 6:578-590.

Zhao C, Fancy SP, Magy L, Urwin JE, Franklin RJ (2005) Stem cells, progenitors and myelin repair. J Anat 207:251-258. 